\title{
Cortical plasticity in patients with Parkinson's disease a window for therapeutic non-invasive neuromodulation
}

\author{
A. QUARTARONE', V. RIZZO', C. TERRANOVA', D. BRUSCHETTA², D. MILARDI ${ }^{2,3}$, \\ P. GIRLANDA', M.F. GHILARDI ${ }^{4}$ \\ ${ }^{1}$ Department of Neurosciences, Psychiatry and Anaesthesiology, University of Messina, Italy; \\ 2 IRCCS Centro Neurolesi Bonino-Pulejo, Messina, Italy; \\ ${ }^{3}$ Department of Biomedical Science and Morphological and Functional Images \\ (E.M., A.A., F.G., D.M., G.P.A., M.G.), University of Messina, Italy; \\ ${ }^{4}$ Dept. of Physiology, Pharmacology \& Neuroscience, CUNY Medical School, New York, NY, USA
}

\begin{abstract}
A B S T R A C T
Several evidences in animal models have consistently an alteration of cortico-striatal plasticity, which is related to the degeneration of the substantia nigra. An alteration of plasticity have also been reported in humans by recording evoked field potentials in the substantia nigra pars reticulata of PD patients undergoing subthalamic nucleus (STN) stimulation where high-frequency $(H F)$ in the OFF state did not induce a lasting change in field potential amplitude in the substantia nigra. In addition protocols of non-invasive brain stimulation, such as paired associative stimulation (PAS) and theta-burst stimulation (TBS), can be used to investigate cortical plasticity of the human primary motor cortex. Despite data reported in literature are apparently controversial with some studies showing a reduced or increased or even normal LTP and LTD like plasticity, recent evidences suggest the hypothesis that these different patterns of cortical plasticity likely depend on the stage of the disease and on the concomitant administration of L-DOPA. The current review will provide an up-to-date of these issues on cortical plasticity in PD discussing the clinical implications in rehabilitation. In addition in the last section we will review the state of art of non invasive neuromodulation as adjuvant treatment in the advanced stage of the disease.
\end{abstract}

Key words

Motor cortex $\bullet$ Transcranial magnetic stimulation $\bullet$ Cortical plasticity $\bullet$ LTP

\section{Introduction}

Plasticity refers to the property of neurons to modify its structure and function changing the effectiveness in neural circuits in response to different stimuli (Castren et al., 2013). These changes may occur at different levels (neuronal, synaptic, protein, or genomic structure) and are defined as synaptic plasticity (Cooke and Bliss 2013).

The mechanisms involved encompass the recruitment of pathways that are functionally homologous but located in different anatomical areas and the reinforcement of existing synaptic connections, dendritic arborization, and synaptogenesis (Bliss et al., 1973; Feldman et al., 2012). The modulation of synaptic efficacy such as long term potentiation (LTP) and long term depression (LTD) play a central role for adaptive motor control and the formation of procedural memory, as demonstrated in animal models studies (Malenka et al., 2004).

Parkinson's disease is a progressive neurodegenerative disorder characterized by alterations of the basal 
ganglia (BG) thalamocortical networks, primarily due to degeneration of nigrostriatal dopaminergic neurons (Lang et al., 1998).

These subcortical alterations may in turn lead to plastic changes in primary motor cortex (M1), which may compensate for the slow progression of Parkinson's disease (Huang and Rothwell, 2003). In this context, an important point is to determine how much of the clinical picture is the result of the disease itself and how much comes from the plastic reorganization within cortical brain circuits located at distance from the primary site of damage in the BG. In particular, imaging studies have revealed that in patients with $\mathrm{PD}$, the supplementary motor area (SMA) and especially the rostral portion, are less active than in normal subjects (Playford et al., 1992). Decreased activation of this area is paralleled by increased activation in premotor and primary motor cortices (Samuel et al., 1997; Sabatini et al., 2000), which may play a compensatory role. In keeping with this hypothesis, Buhmann and coworkers have suggested that this compensation is a gradual process developing over several years (Buhmann et al., 2003). In fact, in a functional magnetic resonance imaging (fMRI) study in de novo hemi-Parkinsonian patients, they found only a decreased activation of both SMA and primary motor cortex contralaterally to the affected side of the body. Therefore, it is likely that the reorganisation seen in the later stage of the disease takes months or even years to develop.

A compensatory plastic reorganization occurs also at the subcortical level: indeed, in early PD, degeneration of the nigrostriatal dopamine neurons is counterbalanced by an increased turnover in the surviving neurons of the pathway (Morrish et al., 1998). Also, there is an enhanced function of the dopamine nigropallidal projections that may serve to maintain a normal pallidal output in early PD (Whone et al., 2003). Although it is generally regarded as an adaptive process, neural plasticity can also be maladaptive (Quartarone et al., 2006). For example, excessive exercise can trigger maladaptive plasticity and produce focal dystonia by leading to inappropriate associations between inputs and outputs of the motor areas causing errors in selecting muscles used in voluntary movement (Quartarone and Hallett, 2013). In certain cases, maladaptive plasticity may be triggered by other factors, such as chronic drug therapy: several lines of evidence suggest that hyperkinetic disorders such as levodopa-induced dyskinesias (LIDs) and tardive dyskinesias are caused by maladaptive plasticity (Cenci et al., 2007; Quartarone et al., 2006; Teo et al., 2012). Aim of the present paper is to review the impact of plasticity on the clinical picture of PD and how non-invasive brain stimulation techniques may modulate cortical plasticity as an adjuvant treatment in PD.

\section{Primary motor cortex plasticity and non-invasive neuromodulation}

Primary motor cortex (M1) is widely connected with a large number of areas including the parietal lobe, pre-motor cortex, supplementary motor area, BG and the cerebellum. This complex network guarantees motor performance and motor learning (Ito et al., 2002). M1 contains a somatotopically-arranged representation of muscle synergies. These motor maps may be shaped and re-shaped by changing the weight of neural connections. The plastic changes in cortical map are thought to occur through a massive reorganization of cortico-cortical horizontal connectivity mediated by changes in synaptic efficacy involving LTP and LTD (Rioult and Pedotti, 2000). The behavioural results of task-specific modification in spatial and temporal organization of muscle synergies at the cortical level include for instance the smooth and accurate execution of movement sequences (Hammond, 2002).

The dynamic reorganization of motor maps during the acquisition of motor skills can be demonstrated with transcranial magnetic stimulation (TMS) techniques or functional neuroimaging (Siebner et al., 2003). TMS can also be used to induce LTP- and LTD-like changes in the brain and can be employed as adjuvant treatment of movement disorders (Chen et al., 2009). TMS protocols that increase cortical excitability are defined as LTP-like protocols, while those that decrease cortical excitability are referred to as LTD-like protocols (Quartarone et al., 2006). For instance, intermittent theta burst stimulation (iTBS), high frequency rTMS (HF-rTMS) and paired associative stimulation $25 \mathrm{~ms}$ (PAS25) are considered LTP-like protocols; continuous thetaburst stimulation (cTBS), low frequency rTMS (below $1 \mathrm{~Hz}$ ) and PAS $10 \mathrm{~ms}$ are considered LTD- 
like protocols. Transcranial direct current stimulation (tDCS) has also been used to induce plasticity in M1. Anodal cortical tDCS typically induces LTP-like changes, while cathodal tDCS induces LTD-like effects (Nitsche et al., 2001). Protocols interacting on different sets of synapses such as PAS, which targets sensory and motor synapses, induce the so-called "heterosynaptic plasticity". This is different from "homosynaptic plasticity" that is induced by stimulating repeatedly the same set of synaptic connections and whose after-effects depend on the frequency of stimulation (rTMS and TBS) (Christiakova et al., 2009).

\section{Cortical plasticity in Parkinson's disease}

Animal models of PD have consistently shown an alteration of cortico-striatal plasticity (Calabresi et al., 1992), a direct link between impairment of neuroplasticity and the degeneration of the substantia nigra (Calabresi et al., 2007), and the restoration of LTP expression with dopamine treatment (Picconi et al., 2003). Alteration of synaptic plasticity within BG circuits has also been demonstrated in vivo in patients with PD. Indeed, Prescott and co-workers recorded evoked field potentials in the substantia nigra pars reticulata of patients undergoing subthalamic nucleus (STN) stimulation. In these patients, high-frequency (HF) stimulation of STN in the OFF state did not induce a lasting change in field potential amplitude in the substantia nigra. However, the administration of levodopa potentiated the field potential amplitudes (LTP), supporting the hypothesis of DOPA-dependent impaired LTP mechanisms in the BG of PD (Prescott et al., 2009).

Several TMS studies have also revealed impaired plasticity at level of primary motor cortex depending on age, disease duration, side of involvement, dopaminergic medications and the type of protocol employed (Udupa et al., 2013). PAS, probably the most used protocol, takes advantage of the principles of associative plasticity by repeatedly coupling a peripheral afferent input from the median nerve with a cortical TMS pulse applied over M1 with inter-stimulus intervals from 10-25 ms (Stefan et al., 2001; Quartarone et al., 2003). Changes in motor cortex excitability induced by PAS occur rapidly, last for at least 60 minutes, are topographically spe- cific, and are blocked by dextromethorphan (NMDA receptor antagonist) or nimodipine (L-type voltagegated channel antagonist) (Stefan et al., 2002). Early studies in patients with PD have reported abnormally reduced responses to PAS compared to healthy subjects, thus pointing to a decreased cortical associative plasticity (Morgante et al., 2006; Ueki et al., 2006). Dopaminergic medications modulate the altered plasticity in PD (Morgante et al., 2006; Ueki et al., 2006). This restoration correlates with decreased plasticity and disease severity as measured by Unified Parkinson's Disease Rating Scale (UPDRS) scores (Ueki et al., 2006). Furthermore, only PD patients without dyskinesias showed restoration of M1 plasticity by levodopa (Morgante et al., 2006).

Slightly different results have been reported with a different TMS-technique, TBS. In particular, Suppa and co-workers have shown that iTBS, which usually induces LTP, cannot induce any effect in PD, both $\mathrm{OFF}$ and $\mathrm{ON}$ dopaminergic therapy, as well as with and without levodopa induced dyskinesias (Eggers et al., 2010; Suppa et al., 2011). Similar results were obtained by Kishore and associates in de novo patients: iTBS and cTBS (which induces LTD) did not induce any effect and levodopa did not revert plasticity back to normal levels (Kishore et al., 2012). The discrepancy with previous results may be due to the different stages of disease of patients recruited in the different studies. In a subsequent study, Kishore and colleagues tested in a more systematic way the effects of different TBS protocols in both OFF and ON levodopa in different groups of advanced PD patients. Patients were categorized according to their motor response to levodopa into stable responders, fluctuating nondyskinetics and fluctuating dyskinetics. The OFF stable responders showed both LTP and LTD after TBS; fluctuating non-dyskinetics had LTP but no LTD, while fluctuating dyskinetics lost both LTP and LTD. It is likely that cortical LTP-like and LTD-like plasticity phenomena rely on long-term levodopa effects. Therefore it is likely that the gradual reduction of long-term levodopa effects on plasticity, particularly for LTD may predispose to the development of motor complications such as levodopa-induced dyskinesias (LIDs) as disease progresses (Kishore et al., 2013). Similar results have been reported in PD patients without 
LIDs: they had normal LTP and LTD when they were under full levodopa dose but there was no LTP when they were on half dose (Huang et al., 2011). In addition, in patients with LID, LTP was achieved just with half a dose even if they were unresponsive to depotentation (Huang et al., 2011). In contrast with those results, two studies have reported an exaggerated plasticity on the less affected side in drug-naïve patients (Kojovic et al., 2012; Bagnato et al., 2006) and in OFF conditions, which was normalized by dopaminergic medications (Bagnato et al., 2006). It is interesting to note that this exaggerated plasticity observed on the less affected side was associated with a less severe clinical involvement. Interestingly, exaggerated plasticity has also been observed in tremulous patients with scan without evidence of dopaminergic deficit (Schwingenschuh et al., 2010): alterations of cortical plasticity in these patients are reminiscent of those in patients with focal dystonia (Quartarone et al., 2006). Are these alterations of cortical plasticity related to the disease itself or are they induced by levodopa treatment? In a recent study, Kacar and coworkers compared responses to facilitatory PAS in two cohorts of patients with advanced PD: one included chronically and optimally treated patients while the other included patients with advanced PD who had never been treated with levodopa. Again, facilitatory responses to PAS were reduced in both patient cohorts when compared with healthy subjects. Therefore, the reduction of cortical plasticity seems to occur independently of chronic exposure to levodopa, thus representing a primary feature of the PD (Kacar et al., 2013).

Overall these results of studies allows us to put forward some interesting points that may have clinical and rehabilitative implications:

1. Cortical plasticity, as tested with TMS techniques, is severely impaired very early in the course of PD;

2. The response of the motor cortex in PD may vary according to the disease progression and the onset of motor complications;

3. Plasticity alterations do not always respond to dopamine administrations;

4. The reactivity of motor cortex after these plasticity protocols strictly follows the pattern of clinical improvement induced by dopamine administration (Kishore et al., 2012).

\section{Clinical relevance of impaired plasticity in Parkinson's disease}

Unfortunately, PD can only partially be controlled by pharmacological and surgical treatment and in the advanced stages of the disease, there is no rescue therapy to treat mobility and postural problems. Therefore motor rehabilitation could be a valid strategy to improve quality of life and to train patients to learn useful compensatory motor strategies. Rehabilitative practice is largely dependent on the efficiency of motor learning and plasticity phenomena within the sensory-motor circuit. The alteration of motor and sensory motor plasticity revealed in all neurophysiological and behavioural studies suggest that PD patients may have difficulties in the acquisition of new abilities. Indeed, despite patients with PD are able to improve their motor performances through practice, the amount and persistence of clinical benefits are uncertain. Both implicit (or procedural) and explicit (or declarative) motor learning has been investigated in PD patients with serial reaction time paradigms and reaching movements (Nakamura et al 2001). Overall, the results of these studies have revealed an early impairment of explicit learning, while implicit motor learning is relatively preserved (Ghilardi et al., 2003). In addition, consolidation and retention of a given task is defective in PD and is not fully related to dopaminergic treatment (Marinelli et al., 2009; Isaias et al., 2011; Bedard and Sanes, 2011). Moreover, levodopa can worsen the acquisition of new motor sequences (Argyelan et al., 2008). This is in keeping with functional imaging studies where levodopa increases motor-related activation while a decrease is observed during the working memory task (Mattay et al., 2002). On the other hand, motor sequence learning is improved by deep brain stimulation of pallidum (Fukuda et al., 2002). Therefore, the knowledge of mechanisms underlying plasticity, as tested with TMS and related techniques and motor learning, are important starting points in order to conceive more effective rehabilitation protocols in PD patients.

It is worthy to note that we recently used repetitive transcranial magnetic stimulation in PD for the purpose of improving visuo-motor retention in $\mathrm{PD}$. A group of patients with PD was tested in two two-day sessions, separated by one week (rTMS and sham session). The first day of each session, they learned 
to adapt their movements to a step-wise $60^{\circ}$ visual rotation. Immediately after the task, either real $5 \mathrm{~Hz}-\mathrm{rTMS}$ or sham stimulation was applied over the right posterior parietal cortex (P6). In patients with PD, adaptation achieved at the end of training was comparable in the sham and rTMS sessions and was similar to that of a group of age-matched controls. However, retention indices tested on the following day were significantly lower in the sham compared to the rTMS session in which retention indices were restored to the level of the controls. Importantly, reaction and movement time as well as other kinematic measures were the same in the rTMS and sham sessions. These results suggest that rTMS applied after the acquisition of a motor skill over specific areas involved in this process might enhance skill retention in PD (Moisello et al., 2014).

\section{Non invasive brain stimulation: a valuable therapeutic window in PD}

In the previous sections we showed that non-invasive brain stimulation can be used to probe cortical plasticity in PD. Here we review the evidence that rTMS might improve function in parts of the brain that are functioning sub-optimally after injury or in chronic diseases such as PD. In the past years, many studies have used techniques such as rTMS in order to reduce motor impairment in $\mathrm{PD}$. These are based on the fact that rTMS produces after-effects that outlast the period of stimulation when applied repeatedly over several weeks. The nature of these after-effects depends on the number, intensity and frequency of stimulation pulses. For example, stimulation at frequencies higher than $1 \mathrm{~Hz}$ tends to increase motor cortex excitability (Quartarone et al., 2005), whereas lower frequencies rTMS can transiently depress cortical excitability (Chen et al., 2001). Several randomized controlled trials used rTMS to treat the PD motor symptoms (see for a review Elahi et al., 2009). However, the results of these studies are difficult to interpret for major limiting factors, such as the rather small sample size, heterogeneous patient profile (various pharmacological treatment, disease duration, severity, and type of motor symptoms) and the large variety of cortical targets. In general, these factors make the emergence of consensus for any stimulation procedures extremely difficult.
More in detail, some controlled studies in PD patients have demonstrated the therapeutic value of repeated sessions of high frequency rTMS (ranging from $5 \mathrm{~Hz}$ to $25 \mathrm{~Hz}$ ) targeting $\mathrm{M} 1$ hand representation with a global improvement of UPDRS part III motor scores, especially regarding movement speed or also gait velocity (Siebner et al., 1999; Lefaucher et al., 2004; Kedhr et al., 2006). Interestingly, highfrequency $5 \mathrm{~Hz}$ rTMS over leg area followed by 30 min of treadmill training over four weeks resulted in an increased walking speed (Mak et al., 2013).

Alternative target to M1 has been the supplementary motor area (SMA). One study showed that $5 \mathrm{~Hz}$ rTMS once a week for eight weeks induced some improvement of the global UPDRS score (Hamada et al., 2008). However, only a mild improvement in bradykinesia was reported in a subsequent study by the same group (Hamada et al., 2009). Indeed, such an improvement could have been related to an increase in dopamine release, but also to possible placebo effects (Khedr et al., 2007), that are common in PD. The stimulation targets to treat levodopa induced dyskinesias have been SMA using low frequency stimulation $(1 \mathrm{~Hz})$ protocols (Koch et al., 2005; Brusa et al., 2006) and the cerebellum, exploiting the possibility of modulating cerebello-thalamo-cortical circuits (Kishore et al., 2013). Indeed, reduction of peak-dose dyskinesia up to four weeks has been described following repeated sessions of excitabilitydecreasing cTBS $(50 \mathrm{~Hz})$ bilaterally delivered to the lateral cerebellum (Koch et al., 2009).

Overall, a review of the current literature suggests that high-frequency rTMS might be a promising treatment of motor symptoms in PD. However, first, as we discussed, the results of the different studies are still ambiguous. Second, there is no direct evidence that the clinical improvement induced by stimulation is related to restoration of the altered plasticity mechanisms described above. Third, other issues needs to be clarified, such as the optimal stimulation parameters, how the different stages of PD affect the response to rTMS, and the effects of rTMS on other aspects of the disease, such as gait, cognition, and memory. Fourth, the effects of the different protocols might be stratified according to the different profile of LTP-like and LTD-like alterations probed with TMS. This could allows for the identification of responders or non-responder to a specific stimulation protocol. 
Future studies are needed to address the role of stimulation techniques in the context of motor and cognitive rehabilitation strategies in PD.

\section{References}

Argyelan M., Carbon M., Ghilardi M.F., Feigin A., Mattis P., Tang C., Dhawan V., Eidelberg D. Dopaminergic suppression of brain deactivation responses during sequence learning. J. Neurosci, 28: 10687-10695, 2008.

Bagnato S., Agostino R., Modugno N., Quartarone A., Berardelli A. Plasticity of the motor cortex in Parkinson's disease patients on and off therapy. Mov. Disord., 21: 639-645, 2006.

Bédard P. and Sanes J.N. Basal ganglia-dependent processes in recalling learned visual-motor adaptations. Exp. Brain Res., 209: 385-393, 2011.

Bliss T.V. and Gardner-Medwin A.R. Long-lasting potentiation of synaptic transmission in the dentate area of the unanaestetized rabbit following stimulation of the perforant path. J. Physiol., 232: 357-374, 1973.

Brusa L., Versace V., Koch G., Iani C., Stanzione P., Bernardi G., Centonze D. Low frequency rTMS of the SMA transiently ameliorates peak-dose LID in Parkinson's disease. Clin. Neurophysiol., 117: 1917-1921, 2006.

Buhmann C., Glauche V., Stürenburg H.J., Oechsner M., Weiller C., Büchel C. Pharmacologically modulated fMRI. Cortical responsiveness to levodopa in drug-naive hemiparkinsonian patients. Brain, 126: 451-461, 2003.

Calabresi P., Maj R., Pisani A., Mercuri N.B., Bernardi G. Long-term synaptic depression in the striatum: physiological and pharmacological characterization. J. Neurosci., 12: 4224-4233, 1992.

Calabresi P., Picconi B., Tozzi A., Di Filippo M. Dopamine-mediated regulation of corticostriatal synaptic plasticity. Trends Neurosci., 3: 211-292, 2007.

Castrén E. and Hen R. Neuronal plasticity and antidepressant actions. Trends Neurosci., 36: 259-267, 2013.

Cenci M.A. L-DOPA-induced dyskinesia: cellular mechanisms and approaches to treatment. Parkinsonism Relat. Disord., 3: 63-77, 2013.

Chen R. and Seitz R.J. Changing cortical excitability with low-frequency magnetic stimulation. Neurology, 57: 379-380, 2001.
Chen R. and Udupa K. Measurement and modulation of plasticity of the motor system in humans using transcranial magnetic stimulation. Motor Control., 13: 442-453, 2009.

Chistiakova M. and Volgushev M. Heterosynaptic plasticity in the neocortex. Exp. Brain Res., 199: 377-390, 2009.

Cooke S.F. and Bliss T.V. Plasticity in the human central nervous system. Brain, 129: 1659-1673, 2006.

Eggers C., Fink G.R., Nowak D.A. Theta burst stimulation over the primary motor cortex does not induce cortical plasticity in Parkinson's disease. $J$. Neurol., 257: 1669-1674, 2010.

Elahi B. and Chen R. Effect of transcranial magnetic stimulation on Parkinson motor function. Systematic review of controlled clinical trials. Mov. Disord., 24: 357-363, 2009.

Feldman D.E. The spike-timing dependence of plasticity. Neuron, 75: 556-571, 2012.

Fukuda M., Ghilardi M.F., Carbon M., Dhawan V., Ma Y., Feigin A., Mentis M.J., Ghez C., Eidelberg D. Pallidal stimulation for parkinsonism: improved brain activation during sequence learning. Ann. Neurol., 52: 144-152, 2002.

Ghilardi M.F., Eidelberg D., Silvestri G., Ghez C. The differential effect of PD and normal aging on early explicit sequence learning. Neurology, 60 : 1313-1319, 2003.

Hamada M., Ugawa Y., Tsuji S. High-frequency rTMS over the supplementary motor area for treatment of Parkinson's disease Effectiveness of rTMS on Parkinson's Disease Study Group, Japan. Mov. Disord., 23: 1524-1531, 2008.

Hamada M., Ugawa Y., Tsuji S. High-frequency rTMS over the supplementary motor area improves bradykinesia in Parkinson's disease: subanalysis of double-blind sham-controlled study. Effectiveness of rTMS on Parkinson's Disease Study Group, Japan. J. Neurol. Sci., 287: 143-146, 2009.

Hammond G. Correlates of human handedness in primary motor cortex: a review and hypothesis. Neurosci. Biobehav. Rev., 26: 285-292, 2002.

Huang Y.Z., Rothwell J.C., Lu C.S., Chuang W.L., Chen R.S. Abnormal bidirectional plasticity-like effects in Parkinson's disease. Brain, 134: 23122320, 2011.

Isaias I.U., Moisello C., Marotta G., Schiavella M., Canesi M., Perfetti B., Cavallari P., Pezzoli G., Ghilardi M.F. Dopaminergic striatal innervation predicts interlimb transfer of a visuomotor skill. $J$. Neurosci., 31: 14458-14462, 2011. 
Ito M. Historical review of the significance of the cerebellum and the role of Purkinje cells in motor learning. Ann. NY Acad. Sci., 978: 273-288, 2002.

Kačar A., Filipović S.R., Kresojević N., Milanović S.D., Ljubisavljević M., Kostić V.S., Rothwell J.C. History of exposure to dopaminergic medication does not affect motor cortex plasticity and excitability in Parkinson's disease. Clin. Neurophysiol., 124: 697-707, 2013.

Khedr E.M., Rothwell J.C., Shawky O.A., Ahmed M.A., Foly N., Hamdy A. Dopamine levels after repetitive transcranial magnetic stimulation of motor cortex in patients with Parkinson's disease: preliminary results. Mov. Disord., 22: 1046-1050, 2007.

Kishore A., Joseph T., Velayudhan B., Popa T., Meunier S. Early, severe and bilateral loss of LTP and LTD-like plasticity in motor cortex (M1) in de novo Parkinson's disease. Clin. Neurophysiol., 123: 822-828, 2012.

Kishore A., Popa T., Balachandran A., Chandran S., Pradeep S., Backer F., Krishnan S., Meunier S. Cerebellar sensory processing alterations impact motor cortical plasticity in Parkinson's disease: clues from dyskinetic patients. Cereb. Cortex, 24: 2055-2067, 2014.

Koch G., Brusa L., Caltagirone C., Peppe A., Oliveri M., Stanzione P., Centonze D. rTMS of supplementary motor area modulates therapy-induced dyskinesias in Parkinson disease. Neurology, 65 : 623-625, 2005.

Koch G., Brusa L., Carrillo F., Lo Gerfo E., Torriero S., Oliveri M., Mir P., Caltagirone C., Stanzione P. Cerebellar magnetic stimulation decreases levodopa-induced dyskinesias in Parkinson disease. Neurology, 73: 113-119, 2009.

Kojovic M., Bologna M., Kassavetis P., Murase N., Palomar F.J., Berardelli A., Rothwell J.C., Edwards M.J., Bhatia K.P. Functional reorganization of sensorimotor cortex in early Parkinson disease. Neurology, 78: 1441-1448, 2012.

Lang A.E. and Lozano A.M.. Parkinson's disease. First of two parts. N. Engl. J. Med., 339: 10441053, 1998.

Lefaucheur J.P. Transcranial magnetic stimulation in the management of pain. Suppl. Clin. Neurophysiol., 57: 737-748, 2004.

Mak M.K. Repetitive transcranial magnetic stimulation combined with treadmill training can modulate corticomotor inhibition and improve walking performance in people with Parkinson's disease. $J$. Physiother., 59: 128, 2013.
Malenka R.C. and Bear M.F. LTP and LTD: an embarrassment of riches. Neuron, 44: 5-21, 2004.

Marinelli L., Crupi D., Di Rocco A., Bove M., Eidelberg D., Abbruzzese G., Ghilardi M.F. Learning and consolidation of visuo-motor adaptation in Parkinson's disease. Parkinsonism Relat. Disord., 15: 6-11, 2009.

Mattay V.S., Tessitore A., Callicott J.H., Bertolino A., Goldberg T.E., Chase T.N., Hyde T.M., Weinberger D.R. Dopaminergic modulation of cortical function in patients with Parkinson's disease. Ann. Neurol., 5: 156-164, 2002.

Morgante F., Espay A.J., Gunraj C., Lang A.E., Chen R. Motor cortex plasticity in Parkinson's disease and levodopa-induced dyskinesias. Brain, 129: 1059-1069, 2006.

Morrish P.K., Rakshi J.S., Bailey D.L., Sawle G.V., Brooks D.J. Measuring the rate of progression and estimating the preclinical period of Parkinson's disease with [18F]dopa PET. J. Neurol. Neurosurg. Psychiatry, 64: 314-319, 1998.

Nakamura T., Ghilardi M.F., Mentis M., Dhawan V., Fukuda M., Hacking A., Moeller J.R., Ghez C., Eidelberg D. Functional networks in motor sequence learning: abnormal topographies in Parkinson's disease. Hum. Brain Mapp., 12: 42-60, 2001.

Nitsche M.A. and Paulus W. Sustained excitability elevations induced by transcranial DC motor cortex stimulation in humans. Neurology, 57: 18991901, 2001.

Picconi B., Centonze D., Håkansson K., Bernardi G., Greengard P., Fisone G., Cenci M.A., Calabresi P. Loss of bidirectional striatal synaptic plasticity in L-DOPA-induced dyskinesia. Nat. Neurosci., 6: 501-506, 2003.

Playford E.D., Jenkins I.H., Passingham R.E., Nutt J., Frackowiak R.S., Brooks D.J. Impaired mesial frontal and putamen activation in Parkinson's disease: a positron emission tomography study. Ann. Neurol., 32: 151-161, 1992.

Prescott I.A., Dostrovsky J.O., Moro E., Hodaie M., Lozano A.M., Hutchison W.D. Levodopa enhances synaptic plasticity in the substantia nigra pars reticulata of Parkinson's disease patients. Brain, 132: 309-318, 2009.

Quartarone A. and Hallett M. Emerging concepts in the physiological basis of dystonia. Mov. Disord., 28: 958-967, 2013.

Quartarone A., Bagnato S., Rizzo V., Siebner H.R., Dattola V., Scalfari A., Morgante F., Battaglia F., Romano M., Girlanda P. Abnormal associative 
plasticity of the human motor cortex in writer's cramp. Brain, 126: 2586-2596, 2003.

Quartarone A., Bagnato S., Rizzo V., Morgante F., Sant'angelo A., Battaglia F., Messina C., Siebner H.R., Girlanda P. Distinct changes in cortical and spinal excitability following high-frequency repetitive TMS to the human motor cortex. Exp. Brain Res., 161: 114-124, 2005.

Quartarone A., Siebner H.R., Rothwell J.C. Taskspecific hand dystonia: can too much plasticity be bad for you? Trends Neurosci., 29: 192-199, 2006.

Rioult-Pedotti M.S., Friedman D., Donoghue J.P. Learning-induced LTP in neocortex. Science, 290: 533-366, 2000.

Rothwell J.C. and Huang Y.Z. Systems-level studies of movement disorders in dystonia and Parkinson's disease. Curr. Opin. Neurobiol., 13: 691-695, 2003.

Huang Y.Z., Rothwell J.C., Lu C.S., Chuang W.L., Chen R.S. Abnormal bidirectional plasticity-like effects in Parkinson's disease. Brain, 134: 23122320, 2011.

Samuel M., Ceballos-Baumann A.O., Turjanski N., Boecker H., Gorospe A., Linazasoro G., Holmes A.P., DeLong M.R., Vitek J.L., Thomas D.G., Quinn N.P., Obeso J.A., Brooks D.J. Pallidotomy in Parkinson's disease increases supplementary motor area and prefrontal activation during performance of volitional movements an H2(15)O PET study. Brain, 120: 1301-1313, 1997.

Sabatini U., Boulanouar K., Fabre N., Martin F., Carel C.., Colonnese C., Bozzao L., Berry I., Montastruc J.L., Chollet F., Rascol O. Cortical motor reorganization in akinetic patients with Parkinson's disease: a functional MRI study. Brain, 123: 394403, 2000.

Schwingenschuh P., Ruge D., Edwards M.J., Terranova C., Katschnig P., Carrillo F., SilveiraMoriyama L., Schneider S.A., Kägi G., Palomar F.J., Talelli P., Dickson J., Lees A.J., Quinn N.,
Mir P., Rothwell J.C., Bhatia K.P. Distinguishing SWEDDs patients with asymmetric resting tremor from Parkinson's disease: a clinical and electrophysiological study. Mov. Disord., 25: 560-569, 2010.

Siebner H.R., Mentschel C., Auer C., Conrad B. Repetitive transcranial magnetic stimulation has a beneficial effect on bradykinesia in Parkinson's disease. Neuroreport, 10: 589-594, 1999.

Siebner H.R. and Rothwell J. Transcranial magnetic stimulation: new insights into representational cortical plasticity. Exp. Brain Res., 148: 1-16 2003.

Stefan K., Kunesch E., Benecke R., Classen J. Effects of riluzole on cortical excitability in patients with amyotrophic lateral sclerosis. Ann. Neurol., 49: 536-539, 2001.

Stefan K., Kunesch E., Benecke R., Cohen L.G., Classen J. Mechanisms of enhancement of human motor cortex excitability induced by interventional paired associative stimulation. J. Physiol., 543: 699-708, 2002.

Suppa A., Marsili L., Belvisi D., Conte A., Iezzi E., Modugno N., Fabbrini G., Berardelli A. Lack of LTP-like plasticity in primary motor cortex in Parkinson's disease. Exp Neurol., 227: 296-301, 2011.

Teo J.T., Edwards M.J., Bhatia K. Tardive dyskinesia is caused by maladaptive synaptic plasticity: a hypothesis. Mov. Disord., 27: 1205-1215, 2012.

Udupa K. and Chen R. Motor cortical plasticity in Parkinson's disease. Front Neurol., 4: 128, 2013.

Ueki Y., Mima T., Kotb M.A., Sawada H., Saiki H., Ikeda A., Begum T., Reza F., Nagamine T., Fukuyama H. Altered plasticity of the human motor cortex in Parkinson's disease. Ann. Neurol., 59: 60-71, 2006.

Whone A.L., Moore R.Y., Piccini P.P., Brooks D.J. Plasticity of the nigropallidal pathway in Parkinson's disease. Ann. Neurol., 53: 206-213, 2003. 